\title{
Dispelling fears over pesticides
}

Pesticides and Human Welfare. Edited by D. L. Gunn and J. G. R. Stevens. Pp. 278. (Oxford University: Oxford and London, 1977.) Hardback £5; paperback $£ 1.75$.

THERE has been a great deal written and said about the alleged disadvantages and dangers of pesticides. This book has been produced in response to this publicity, and is sponsored by seven chemical companies from western Europe, including ICI and Shell from England. The book is intended for laymen and non-specialists, and so discusses, in a lengthy appendix, the meaning of some common technical terms, types of pesticides, their formulation and biological activity. The main text contains three parts, with a total of twenty chapters, each intended to be scientifically accurate, intelligible and authoritative.

There are four chapters in part one. The first three, on the world's food supply (W. R. Furtick), population growth (R. H. Gray), and vector-borne diseases (G. Davidson), introduce the problems that pesticides can help to solve. They combine to illustrate vividly the differences between the industrialised and the developing countries of the world. Data are provided for past and expected future increases in population and food production, It is estimated, for example, that pests consume about $30 \%$ of the potential food production in the developing countries, but they use only $10 \%$ of the world's pesticides, mainly for public health and exported crops. The developing countries have higher rates of population increase, and, just to maintain present standards, their food production will probably need to almost double during the next twenty-five years. The important point is made several times that methods of food production, medical services and birth rates cannot be considered in isolation, but are all components of a society's culture. Thus, the effective use of pesticides in food production implies a relatively sophisticated agricultural industry. The fourth chapter, by D. J. Ansell, explains some of the financial difficulties in increasing food production in the developing countries.

The second part, with ten chapters, provides examples of how pesticides improve crop yields, animal health and food storage. The criterion by which pesticides are judged is their cost against the value of the increment in yield. G. Schumann suggests, particularly from German experience, that farmers get a return of between $1: 3$ and $1: 9$ for pesticides, going in exceptional cases as high as $1: 100$. Several other chapters comment, for specific crops, on the lack of precise figures: the introduction to the chapter on tropical cash crops discusses some of the reasons for this, and concludes that in general when pesticides are used their benefits are so obvious that detailed data are unnecessary. Such calculations, when possible, are based of course on a micro-economic analysis. D. E. Jacobs indicates that realistic cost-benefit analyses for an entire community need computer-aided models. J. P. Hudson touches on another aspect of the same problem when he explains that for commercial orchards, once the current standard spraying regimes have been used, it would be disastrous to stop spraying.

The final part, in six chapters, discusses complications of, and alternatives to, pesticides. It is clear that pest resistance to pesticides (J. R. Busvine) is a continuing major worry. The late J. M. Barnes presents a reassuring account of the hazards to people; the major risk comes from human carelessam not completely convinced that animals will always be adequate indicators of possible chronic toxic effects in man ( $\mathrm{p} 188$ ), but that is a minor quibble. K. Mellanby discusses the "balance of nature" and makes the point that one can never prove that all hazards from pesticides are known. Nevertheless, he sees little cause for ness, not from unexpected toxicity. I

alarm on this score, although I doubt whether we have too much evidence either way for some of the developing countries. D. P. Jones discusses the topical issue of energy costs and gains from pesticides. R. F. Glasser describes some of the controls imposed by governments on the development and use of pesticides, and suggests, as do several other authors, that the increasing costs of research are reducing the amount of research for new pesticides. It would have been interesting to have some comments on the fact that it is becoming more difficult to discover new potential pesticides at the primary screening stage. The final chapter, by D. L. Gunn, discusses alternatives to pesticides and concludes that pesticides will remain of major importance for the foreseeable future.

This book succeeds in its aims. It is clearly written, the few minor misprints causing no problems except for convential (sic) on p212; most of the chapters read convincingly; and the book should inform and stimulate its intended audience. There are some omissions; for example, there are few if any data on quantities of pesticides manufactured and used, and few references to forestry or to herbicides in waterways. The major unanswered query was, where are we going? Clearly, in the short term, we will continue to need and use pesticides. In the long term, if pesticides merely enable us to keep one jump ahead of population increase, for how long will we be able to maintain that state of affairs?

F. Moriarty is a Principal Scientific Officer at Monks Wood Experimental Station, Institute of Terrestrial Ecology, Huntingdon, UK.

\section{Dynamics of atomic liquids}

Atomic Dynamics in Liquids. By N. H. March and M. P. Tosi. Pp. 330. (Macmillan: London, 1976.) £25.

IT is difficult to decide for whom this book is intended. The level of discussion is high and the range covered is very wide. As may be expected the longest chapter is on charged fluids (plasmas, liquid metals and molten saits), a field to which the authors have made important contributions. Apart from the conventional general discussion of the structure and dynamics of pure atomic liquids and binary liquid mixtures there are short specialised chapters on the helium liquids, the liquid surface and on critical phenomena. Few topics are discussed or explained in any detail, and a considerable expertise in theoretical physics is a prerequisite. Consequently this book cannot be recommended to the beginner. On the other hand it will not be very exciting for the expert, except as a reference book.

The material is not well organised and the 